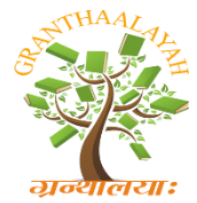

\author{
INTERNATIONAL JOURNAL OF RESEARCH \\ GRANTHAALAYAH \\ A knowledge Repository
}

Science

\title{
FEMTOSECOND LASER PULSE PROPAGATION IN NOVEL FIBER: TELLURITE GLASS CODOPED $\mathrm{ER}^{3+} / \mathrm{TM}^{3+}$
}

\author{
Khelladi. $M^{* 1}$ \\ ${ }^{* 1}$ Department of Electrical and Electronics Engineering, Faculty of Technology - Tlemcen \\ Algeria
}

\begin{abstract}
In this work we investigated propagation of ultrashort laser pulses in Tellurite glass codoped Er3+/Tm3+. We derived a general propagation equation of pulses which includes the linear and nonlinear effects to all orders. We studied in the specific case of Kerr media and obtained an ultrashort pulse propagation equation called a generalized nonlinear Schrödinger equation. The impact of the third order dispersion, the higher-order nonlinear terms self-steepening, and stimulated Raman scattering are explicitly analyzed.
\end{abstract}

Keywords: Tellurite Glass; Rare Earth; Schrodinger Equation; Femtosecond Laser.

Cite This Article: Khelladi. M. (2019). "FEMTOSECOND LASER PULSE PROPAGATION IN NOVEL FIBER: TELLURITE GLASS CODOPED ER3+/TM3+." International Journal of Research - Granthaalayah, 7(1), 96-105. 10.29121/granthaalayah.v7.i1.2019.1039.

\section{Introduction}

Theoretical and experimental research for the propagation process of ultrashort laser pulses (in fs) in a medium have been the subject of intensive research within the last few years $[1,2,4]$. Because of special properties of these pulses, during their propagation in the medium several new effects have been observed in comparison with the propagation process of short pulses (in ps), namely the effects of dispersion and nonlinear effects of higher orders. Under the influence of these effects, we have complicated changes both in amplitude and spectrum of the pulse. It splits into constituents and its spectrum also evolves into several bands which are known as optical shock and self-frequency shift phenomena [1-3].

These effects should be studied in detail for future concrete applications of femtosecond pulses, especially in the domain of optical communication. This formalism is based on the approximate expansion of the nonlinear wave equation, which treats the nonlinear processes involved in the problem as the perturbations. 


\section{Tellutite Glass}

\subsection{Definitions}

Tellurite glass fibers exhibit significant advantages over the fluoride and silicate glass fibers for amplification outside the C-band. The Tellurite glass has comparatively lower phonon energy (780 $\mathrm{cm}^{-1}$ ) than silicate glass, and it exhibits better environmental resistance than fluoride fibers, in addition, Tellurite glass provides a much broader fluorescence spectra and larger rare earth oxide solubility than silicate and fluoride glasses [5,6]. Recently, Tellurite fiber doped $\mathrm{Tm}^{3+}$ for S-band amplification were reported under dual-wavelength pumping (795/1064 nm and 1047/1550 nm) schemes ${ }^{7}$.

The Tellurite glass presents very good optical quality, is stable against atmospheric moisture, it exhibits low optical attenuation from $400 \mathrm{~nm}$ to $5.0 \mu \mathrm{m}$. In recent years, the upconversion of infrared light to visible light by rare earth ion doped glasses has been investigated extensively, due to the possibility of infrared pumped visible lasers and the potential applications in areas such as three-dimensional display, optical data storage, optoelectronics, medical diagnostics, sensor, and undersea optical communication [7.8].

\subsection{Refractive Index}

The refraction index of these glasses is relatively high and depends on the wavelength $(\lambda)$ according to [9]

$$
\mathrm{n}(\lambda) \approx \sqrt{1+\frac{3.24 \lambda^{2}}{\lambda^{2}-193^{2}}}
$$

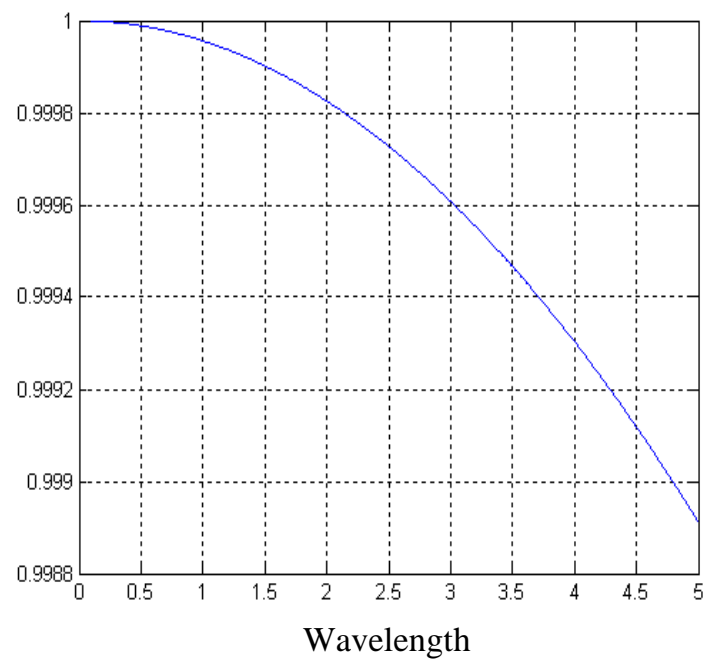

Figure 1: Curves of the refractive index according to the wavelength in the visible field. 


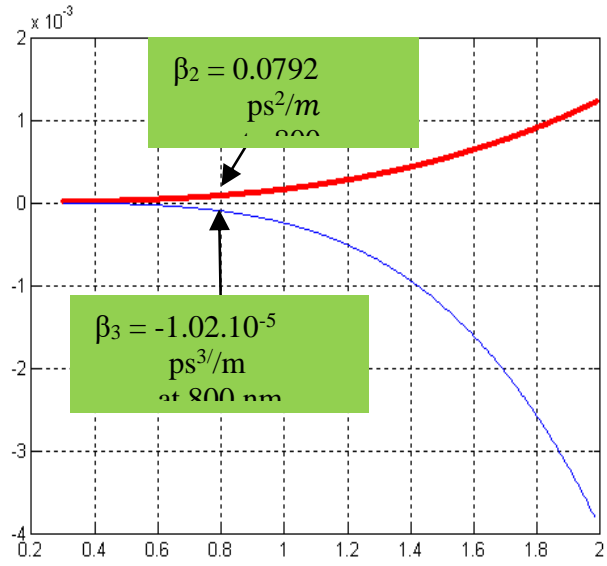

Wavelength

Figure 2: Variation of $\beta_{2}$ and $\beta_{3}$ with wavelength for Tellurite glass Codoped $\mathrm{Er}^{3+} / \mathrm{Tm}^{3+}$.

\section{Propagation Equation for Ultrashort Pulses}

\subsection{General Form of The Pulse Propagation Equation in The Nonlinear Dispersion Medium}

For an understanding of the nonlinear phenomena in optical fibers, it is necessary to consider the theory of electromagnetic wave propagation in dispersive nonlinear media. The Maxwell equations can be used to obtain the following nonlinear wave equation for the electric field.

The study of most nonlinear effects in optical fibers involves the use of short pulses with widths ranging from $\sim 10 n s$ to $10 \mathrm{fs}$. When such optical pulses propagate inside a fiber, both dispersive and nonlinear effects influence their shape and spectrum.

$$
\nabla^{2} E-\frac{1}{C^{2}} \frac{\partial^{2} E}{\partial t^{2}}=\mu_{0} \frac{\partial^{2} P_{L}}{\partial t^{2}}+\mu_{0} \frac{\partial^{2} P_{N L}}{\partial t^{2}}
$$

Where $P_{L}$ and $P_{N L}$ are the linear and nonlinear polarization, respectively.

The electric field $\vec{E}$ is treated as a superposition of monochromatic constituents with different frequencies and wave vectors centered at their central values $w_{0}$ and $\overrightarrow{k_{0}}$. We confine our self only to consider the propagation of the electric field in an arbitrary direction, say $O z$ (usually, chosen as the direction of $\overrightarrow{k_{0}}$ ), so we can write

$$
\vec{E}(r, t)=\frac{1}{2} \vec{x}\left[A(z, t) e^{-i w_{0} t+i k_{0} z}+c . c\right]
$$

The nonlinear Schrodinger equation that governs propagation of optical pulses inside single fiber is given by.

$$
\frac{\partial \mathrm{A}}{\partial \mathrm{z}}+\beta_{1} \frac{\partial \mathrm{A}}{\partial \mathrm{t}}+\frac{\mathrm{i} \beta_{2}}{2} \frac{\partial^{2} \mathrm{~A}}{\partial \mathrm{t}^{2}}+\frac{\alpha}{2} \mathrm{~A}=\mathrm{i} \gamma|\mathrm{A}|^{2} \mathrm{~A},
$$


where the nonlinear parameter $\gamma$ is defined as

$$
\gamma=\frac{\mathrm{n}_{2} \mathrm{w}_{0}}{\mathrm{cA}_{\mathrm{eff}}} \cdot \quad \mathrm{n}_{2}=\frac{3}{8 \mathrm{n}} \operatorname{Re}\left(\chi_{\mathrm{xxxx}}^{(3)}\right)
$$

Eq.(4) describes propagation of picosecond optical pulses in single-mode fibers. It is often referred to as the nonlinear Schrödinger (NLS) equation because it can be reduced to that form under certain conditions. It includes the effects of fiber losses through $\alpha$, of chromatic dispersion through $\beta_{1}$ and $\beta_{2}$, and of fiber nonlinearity through $\gamma$. Briefly, the pulse envelope moves at the group velocity $v_{g} \equiv 1 / \beta_{1}$ while the effects of group-velocity dispersion (GVD) are governed by $\beta_{2}$ [10].

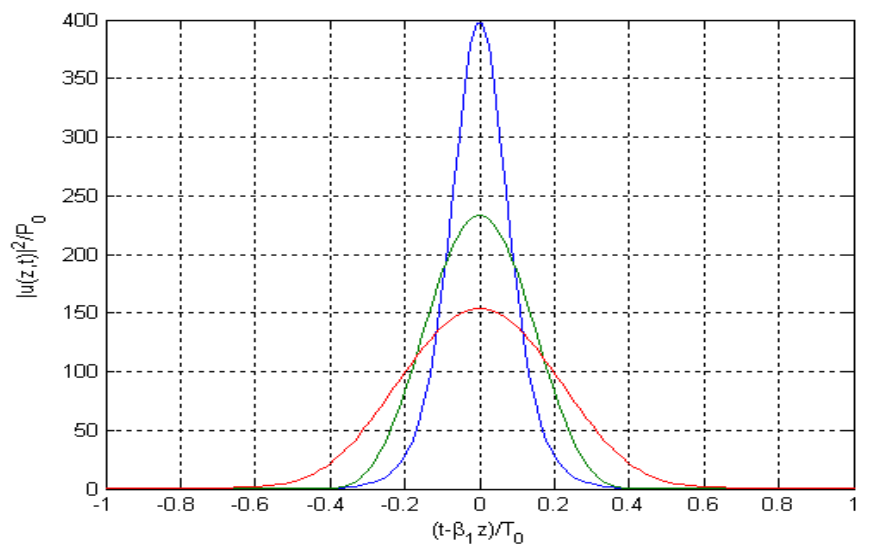

Figure 3: Dispersion-induced broadening of a Gaussian pulse inside Tellurite Glass Codoped

$$
\mathrm{Er}^{3+} / \mathrm{Tm}^{3+} \text { with } \beta_{2}=7.92 e-5 \mathrm{ps}^{2} / \mathrm{m}, \tau_{0}=100 \mathrm{fs}, L_{D} \approx 162 \mathrm{~m}
$$

Figure 3 shows the extent of dispersion-induced broadening for Gaussian pulses by plotting $|U(z, T)|^{2}$ at $z=0,200 L_{D}$ and $400 L_{D}$, for Tellurite Glass Codoped $\mathrm{Er}^{3+} / \mathrm{Tm}^{3+}$. (Comparison with silica fiber see after Agrawal [10] for $z=2$ and $4 L_{D}, \tau_{0}=1 p s, L_{D} \approx 100 \mathrm{~m}$ ).

\subsection{SPM-Induced Spectral Broadening}

An interesting manifestation of the intensity dependence of the refractive index in nonlinear optical media occurs through self-phase modulation (SPM), a phenomenon that leads to spectral broadening

of optical pulses [10]. In terms of the normalized amplitude $U(z, T)$ defined as in Eq.(4), the pulsepropagation become in the limit $\beta_{2}=0$, become

$$
\frac{\partial \mathrm{U}}{\partial \mathrm{z}}=\frac{\mathrm{ie}^{-\alpha \mathrm{z}}}{\mathrm{L}_{\mathrm{NL}}}|\mathrm{U}|^{2} \mathrm{U},
$$

where $A(z, \tau)=\sqrt{P_{0}} \exp (-\alpha z / 2) U(z, \tau)$,

where $\alpha$ accounts for fiber losses. The nonlinear length is defined as

$$
\mathrm{L}_{\mathrm{NL}}=\left(\gamma \mathrm{P}_{0}\right)^{-1} \text {, }
$$



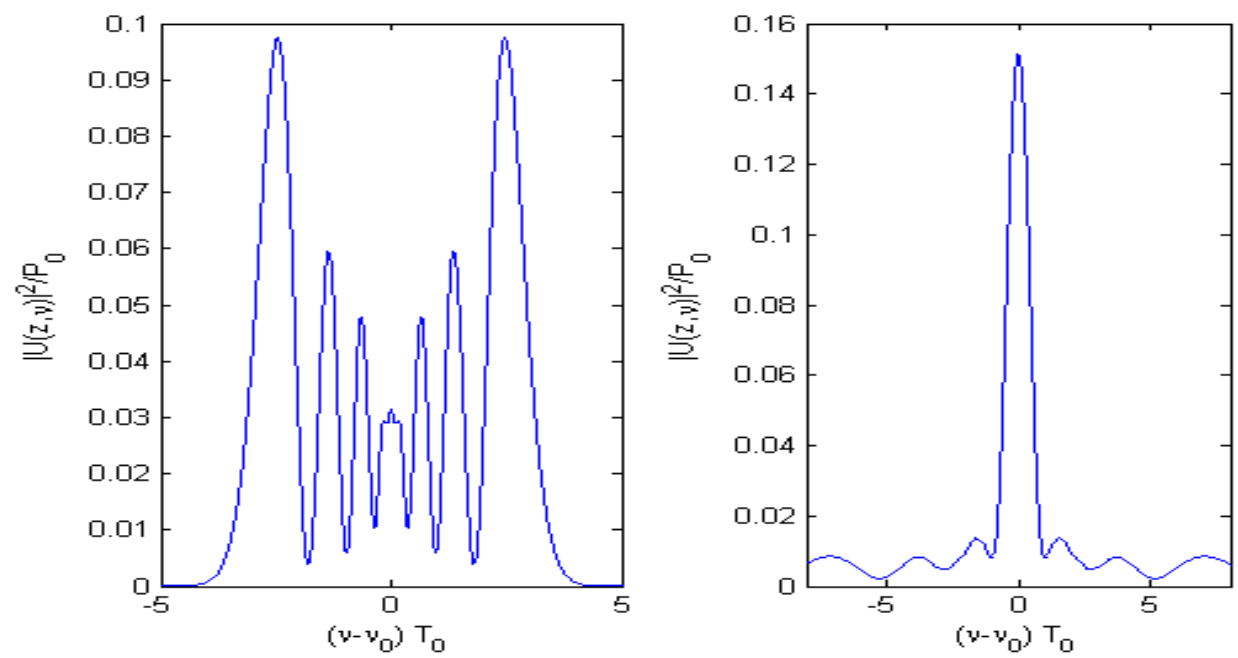

Figure 4: SPM-broadened spectra for an unchirped Gaussian pulse. Spectra are labeled by the maximum nonlinear phase shift $\emptyset_{\max }$. (in the left for Gaussian pulse $\mathrm{m}=0$, and right for superGaussian pulse $\mathrm{m}=3), \tau_{0}=100 \mathrm{fs}, L_{N L}=7.14 \mathrm{~m}$

The most notable feature of Figure4 is that SPM-induced spectral broadening is accompanied by an oscillatory structure covering the entire frequency range. In general, the spectrum consists of many peaks, and the outermost peaks are the most intense. The number of peaks depends on $\emptyset_{\max }$ and increases linearly with it. The origin of the oscillatory structure can be understood by referring to, where the time dependence of the SPM-induced frequency chirp is shown.

Figure. 4 compares the pulse spectra for Gaussian $(m=1)$ and super-Gaussian $(m=3)$ pulses obtained. In both cases, input pulses are assumed to be unchirped $(C=0)$. The fiber length and the peak power are chosen such that $\emptyset_{\max }=50 \pi$. (Comparison with silica fiber see after Agrawal. $\left.\emptyset_{\max }=4.5 \pi, \tau_{0}=1 p s\right)$.

\section{Impact of Dispersion and Higher-Ordernonlinear Effects on The Ultrashort Pulses}

For pulses shorter than $5 \mathrm{ps}$ but wide enough to contain many optical cycles (widths $\gg 10 f s$ ), we can obtain:

$$
\frac{\partial \mathrm{A}}{\partial \mathrm{z}}+\frac{\alpha}{2} \mathrm{~A}+\frac{\mathrm{i} \beta_{2}}{2} \frac{\partial^{2} \mathrm{~A}}{\partial \mathrm{t}^{2}}-\frac{\beta_{3}}{6} \frac{\partial^{3} \mathrm{~A}}{\partial \mathrm{t}^{3}}=\mathrm{i} \gamma\left(|\mathrm{A}|^{2} \mathrm{~A}+\frac{\mathrm{i}}{\mathrm{w}_{0}} \frac{\partial}{\partial \mathrm{T}}\left(|\mathrm{A}|^{2} \mathrm{~A}\right)-\mathrm{T}_{\mathrm{R}} \mathrm{A} \frac{\partial|\mathrm{A}|^{2}}{\partial \mathrm{T}}\right),
$$

The Eq. (8) is the lowest-order approximate form when we consider the higher-order dispersion and nonlinearity effects in the general propagation Eq.(8). It is one of the most useful approximate forms describing the propagation process of the ultrashort pulses, called the generalized nonlinear Schrödinger equation.

Using the new parameters and variables 


$$
\begin{gathered}
\xi=\mathrm{z} / \mathrm{L}_{\mathrm{D}}, \quad \tau=\frac{\mathrm{T}}{\mathrm{T}_{0}}=\frac{\mathrm{t}-\mathrm{z} / \mathrm{v}_{\mathrm{g}}}{\mathrm{T}_{0}} \\
\mathrm{~N}^{2}=\frac{\mathrm{L}_{\mathrm{D}}}{\mathrm{L}_{\mathrm{NL}}} \equiv \frac{\gamma \mathrm{P}_{0} \mathrm{~T}_{0}^{2}}{\left|\beta_{2}\right|} . \quad \mathrm{L}_{\mathrm{D}}^{\prime}=\frac{\mathrm{T}_{0}^{3}}{\left|\beta_{3}\right|} \\
\mathrm{L}_{\mathrm{D}}=\frac{\mathrm{T}_{0}^{2}}{\left|\beta_{2}\right|}, \quad \mathrm{L}_{\mathrm{NL}}=\frac{1}{\gamma \mathrm{P}_{0}} . \\
\delta_{3}=\frac{\beta_{3}}{6\left|\beta_{2}\right| \mathrm{T}_{0}} ; \quad \mathrm{s}=\frac{1}{\mathrm{w}_{0} \mathrm{~T}_{0}} ; \quad \tau_{\mathrm{R}}=\frac{\mathrm{T}_{\mathrm{R}}}{\mathrm{T}_{0}} .
\end{gathered}
$$

where $\tau_{0}$ and $P_{0}$ stand respectively for the time width and the maximal power in the top of the envelope function.

The propagation Eq. (8) for the ultrashort pulses has a more complicated form than the nonlinear Schrödinger equation describing the propagation of the short pulses because it contains the higherorder dispersive and nonlinear terms. The parameters characterizing these effects: $\delta_{3}, s, \tau_{R}$ govern respectively the effects of third order dispersion, self-steepening and the self-shift frequency. From the formulas (9) we see that when $\tau_{0}$ decreases, i.e. the pulse is shorter, the magnitude of these parameters increases, and the higher-order effects should be considered $[11,12]$.

\subsection{Effect of Third-Order Dispersion}

The appropriate propagation equation for the amplitude $A(z, T)$ is obtained from Eq.(8) after setting $\gamma=0 . U(z, T)$ satisfies the following equation:

$$
\mathrm{i} \frac{\partial \mathrm{U}}{\partial \mathrm{z}}=\frac{\beta_{2}}{2} \frac{\partial^{2} \mathrm{U}}{\partial \mathrm{T}^{2}}+\mathrm{i} \frac{\beta_{3}}{6} \frac{\partial^{3} \mathrm{U}}{\partial \mathrm{T}^{3}} .
$$

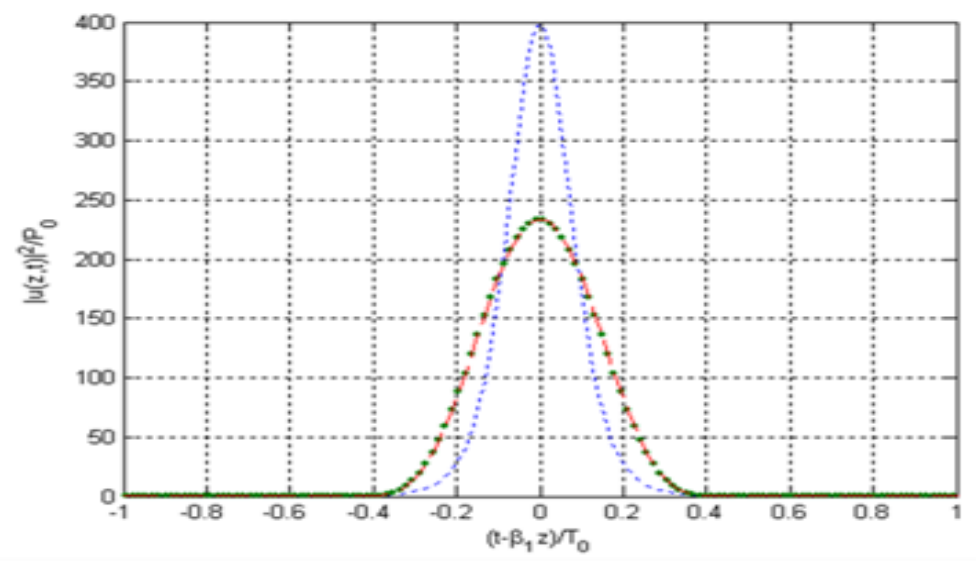

Figure 5: Pulse shapes at $z=200 L_{D}^{\prime}$ of an initially Gaussian pulse at $z=0$ (solid curve) in the presence of $\beta_{2}$ second order dispersion. Dashed curve shows the effect higher order dispersion

$$
\beta_{2} \text { and } \beta_{3} \neq 0, \tau_{0}=100 f s
$$


Figure.5 shows the pulse shapes at $z=200 L_{D}^{\prime}$ for an initially unchirped Gaussian pulse $(C=0)$ for $\beta_{3}=0$ (dotted curve) and for a value of $\beta_{2}=7.92 e-5 p s^{2} / \mathrm{m}$ and $\beta_{3}=-1.02 e-$ $8 \mathrm{ps}^{3} / \mathrm{m}$ (dashed curve). Whereas a Gaussian pulse remains Gaussian when only the $\beta_{2}$ term in Eq (11) contributes to GVD, the TOD do not distorts the pulse because have negligible values. Comparison with silica fiber see after Agrawal [10] $=5 L_{D}^{\prime}, \tau_{0}=1 p s$ ).

Under the influence of TOD both the pulse shape and spectrum change in a complicated way. When the propagation distance is larger the oscillation of the envelope function is stronger, creating a long trailing edge to the later time, and the spectrum is broadened into two sides and splits to the several peaks [12].

\subsection{Self-Steepening}

The phenomenon of self-steepening has been studied extensively. To isolate the effects of selfsteepening governed by the parameter $s$, it is useful to set $\delta_{3}=0$ and $\tau_{R}=0$ in Eq. (8). Pulse evolution inside fibers is then governed by

$$
\mathrm{i} \frac{\partial \mathrm{u}}{\partial \xi}+\frac{1}{2} \frac{\partial^{2} \mathrm{u}}{\partial \tau^{2}}+|\mathrm{u}|^{2} \mathrm{u}+\text { is } \frac{\partial}{\partial \tau}\left(|\mathrm{u}|^{2} \mathrm{u}\right)=0
$$

Self-steepening creates an optical shock on the trailing edge of the pulse in the absence of the GVD effects. This phenomenon is due to the intensity dependence of the group velocity that results in the peak of the pulse moving slower than the wings. The GVD dissipates the shock and smoothes the trailing edge considerably. However, self-steepening would still manifest through a shift of the pulse center.

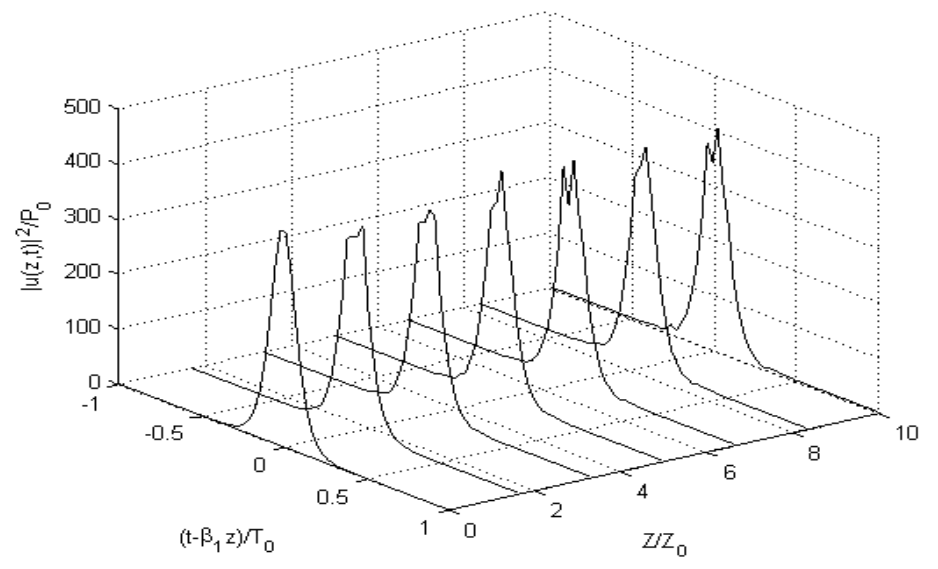

Figure 6: Decay of a second-order soliton $(N=2)$ induced by self-steepening $(s=0.0042)$,

$$
\tau_{0}=100 f s, z=10 \pi / 2
$$

Self-steepening of the pulse leads to the formation of a steep front in the trailing edge of the pulse, resembling the usual shock wave formation. This effect is called the optical shock. The pulse becomes more asymmetric in the propagation and its tail finally breaks up [12]. 
The effect of self-steepening on higher-order solitons is remarkable in that it leads to breakup of such solitons into their constituents, a phenomenon referred to as soliton decay. Figure 9 shows this behavior for a second-order soliton $(N=2)$ using $s=0.0042$ For this relatively large value of $s$, the two solitons have separated from each other within a distance of two soliton periods and continue to move apart with further propagation inside the Tellurite glass. (Comparison with silica fiber see after Agrawal [10], $z=5 \pi / 2, s=0.2, \tau_{0}=1 p s$ ).

A qualitatively similar behavior occurs for smaller values of $s$ except that a longer distance is required for the breakup of solitons. In the absence of self-steepening $(s=0)$, the two solitons form a bound state because both of them propagate at the same speed (the eigenvalues have the same real part). The effect of self-steepening is to break the degeneracy so that the two solitons propagate at different speeds. As a result, they separate from each other, and the separation increases almost linearly with the distance.

\subsection{Intrapulse Raman Scattering}

Intrapulse Raman scattering plays the most important role among the higher-order nonlinear effects. Its effects on solitons are governed by the last term in Eq. (8) and were observed experimentally in 1985. Since then, this higher-order nonlinear effect has been studied extensively. To isolate the effects of intrapulse Raman scattering, it is useful to set $\delta_{3}=0$ and $s=0$ in Eq. (9). Pulse evolution inside fibers is then governed by:

$$
\mathrm{i} \frac{\partial \mathrm{u}}{\partial \xi}+\frac{1}{2} \frac{\partial^{2} \mathrm{u}}{\partial \tau^{2}}+|\mathrm{u}|^{2} \mathrm{u}=\tau_{\mathrm{R}} \mathrm{u} \frac{\partial|\mathrm{u}|^{2}}{\partial \tau}
$$

In the stimulated Raman scattering the Stokes process is more effective than the anti-Stokes process. This fact leads to the so-called self-shift frequency of the pulse.

As a result the spectrum is shifted down to the low frequency region. In other words, the medium "amplifies" the long wavelength parts of the pulse. The pulse losses its energy and changes complicatedly when it enters deeply into medium.

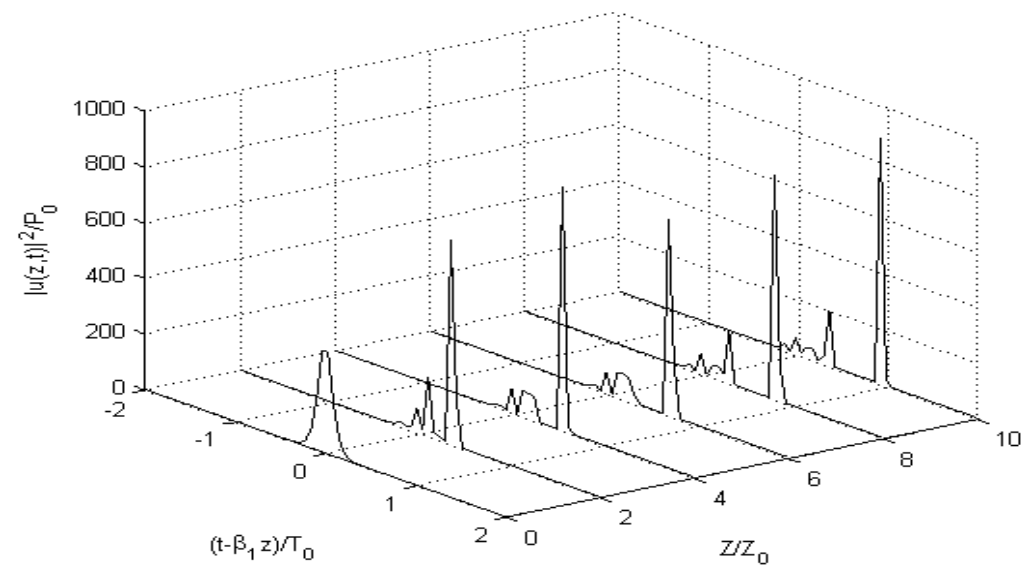

Figure 7: Decay of a second-order soliton $(N=2)$ induced by intrapulse Raman scattering

$$
\left(\tau_{R}=0.01\right) \tau_{0}=100 \mathrm{fs}, z=50 \pi / 2 \text {. }
$$


The effect of intrapulse Raman scattering on higher-order solitons is take a minor role than the case of self-steepening. In particular, even for a large value of $\tau_{R}$ and for the long distance of the propagation, this effect change the form of the pulse relatively. Figure 10 shows such a decay for a second-order soliton $(N=2)$ by solving Eq. (12) numerically with $\tau_{R}=0.01$. (Comparison with silica fiber see after Agrawal ${ }^{11} z=5 \pi / 2$, and $\tau_{0}=1 p s$ ).

\subsection{Generalized Nonlinear Schrodinger Equation}

For the ultrashort pulses with the width $\tau_{0}=100 \mathrm{fs}$ and the carrier wavelength $\lambda_{0}=0.8 \mu \mathrm{m}$ the higher-order parameters in Eqs.(10) during their propagation in the Tellurite glass codoped $\mathrm{Er}^{3+} / \mathrm{Tm}^{3+}$ have the values $\delta_{3} \approx 0.0021, s \approx 0.0042$, and $\tau_{R} \approx 0.01$. These values are smaller than one, so the higher-order effects are considered as the perturbations in comparison with the

Kerr effect.

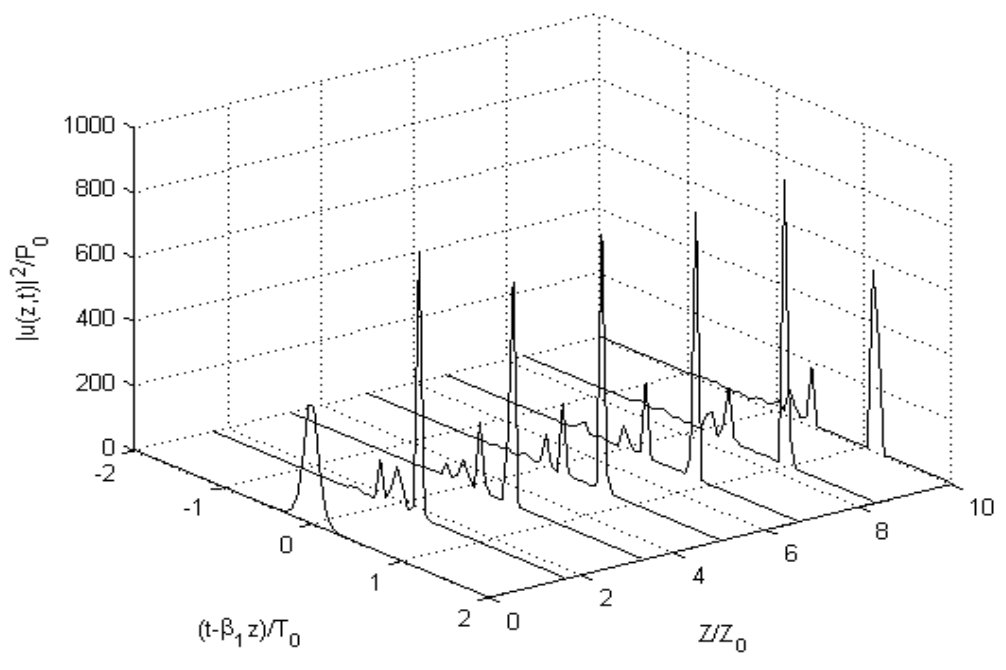

Figure 8: Evolution of pulse shapes with $\beta_{2}=7.92 e-\frac{5 p s^{2}}{m}, \beta_{3}=-1.02 e-8 p s^{3} / m, \gamma=$

$$
0.14, \tau_{0}=100 f s, z=10 \pi / 2
$$

Therefore, when the pulse propagates in a Tellurite glass codoped $\mathrm{Er}^{3+} / \mathrm{Tm}^{3+}$ medium, the selfsteepening effect dominates over the third order dispersion and self-shift frequency for the pulses with the width of hundreds and tens femtoseconds. When $\tau_{0}$ has the value of picoseconds or larger, the values of $\delta_{3}, S$ and $\tau_{R}$ are very small and they can be neglected. The Eq. (8) reduces to the well-known NLS equation for the short pulses.

As successful as Eq. (8) is in modeling the propagation of femtosecond pulses in optical fibers, it is still approximate. Eq. (9) has been used to study numerically how self-steepening affects the evolution of femtosecond optical pulses in optical fibers for Tellurite glass. (Comparison with silica fiber see after Agrawal $z=3 \pi / 2$ and $\left.\tau_{0}=1 p s\right)$. 


\section{Conclusions}

In this paper we derived the Generalized Nonlinear Schrödinger (GNLS) Equation for the propagation process of the ultrashort pulses in the novel fiber like Tellurite glass codoped

$\mathrm{Er}^{3+} / \mathrm{Tm}^{3+}$. The influence of the higher-order dispersive and nonlinear effects, especially the nonlinear effect induced by the self-steepening, have been considered in detail. Because the GNLS equation is strongly nonlinear, the problem of solving it is a difficult task. Until now we have not been able to find any exact analytical solution for this equation in the general case. Several approximate methods of solving it are applied.

Tellurite glass fibers exhibit significant advantages over the fluoride and silicate glass fibers for propagation of femtosecond laser pulse we presented here that this novel material is capable to resolve the problem proposed by self-steepening and intra pulse Raman scattering in silica and fluoride fiber for a long distance and for femtosecond pulse.

\section{References}

[1] R. W. Boyd, Nonlinear Optics, Academic Press Inc., (2003).

[2] U. Bandelow, A. Demircan and M. Kesting. Simulation of Pulse Propagation in Nonlinear Optical Fibers, WIAS, (Bandelow et al. (2003).

[3] Y.S. Kivshar, G. P. Agrawal. Optical Solitons: From Fibers to Photonic Crystals, Academic Press, San Diego, Kivshar, Agrawal, (2003).

[4] C. Headley, and G.P. Agrawal. J. Opt. Soc. Am. B13, 2170-2177 (headley et al. (1996).

[5] S. Shaoxiong, J. Animesh, H. Lihui, and Purushottam. J, "980-nm diode-pumped Tm3+/Yb3+codoped tellurite fiber for S-band amplification," Opt. Lett. (2005).

[6] S. Tanabe. "Properties of Tm3+-doped Tellurite glasses for 1.4-um amplifier," Proc. SPIE 4282, (2001).

[7] L.H. Huang, Liu. X.R, Xu. W, Chen. B.J, and Lin J.L, J. Appl. Phys. 90. (2001).

[8] Man. S.O,. Pun. E.Y, and Chung. P.S., Appl. Phys. Lett. 77.(2000).

[9] E. Rodriguez, A.R.Neves. W.C. Moreira, C. L.César, Er3+-Tm3+ co-doped Tellurite fibers for broadband optical fiber amplifier around $1550 \mathrm{~nm}$ band, Optical Fiber Technology 12 . (2006).

[10] G.P. Agrawal. Nonlinear Fiber optics, optics and phoyonics, (2001).

[11] R.W. Boyd, Nonlinear Optics, Academic Press Inc., (2003).

[12] M. Khelladi, Nonlinear propagation of an ultrashort laser pulse in Titanium Sapphire. Thesis, Tlemcen University- Algeria (2010).

*Corresponding author.

E-mail address:mo.khelladi@ gmail.com 